\title{
三叶因子：从实验室研究到临床医学
}

\author{
杜廷义 ${ }^{1,2,3}$, 张 勇 ${ }^{1,3}$, 张 云 1 ,* \\ (1. 中国科学院和云南省动物模型与人类疾病机理重点实验室, 中国科学院昆明动物研究所, 云南 昆明 650223 ; \\ 2. 云南省第一人民医院 检验科, 云南 昆明 650032；3. 中国科学院研究生院，北京 100049)
}

摘要: 三叶因子 (trefoil factor, TFF) 家族是具有一个或多个三叶因子结构域的蛋白质多肽, 在进化上具有高 度的保守性, 具有耐热、耐酶消化的理化特性。哺乳动物的 TFF 有三个成员(TFF1、TFF2 和 TFF3)。黏膜组织, 如胃肠道和呼吸道黏膜等是 TFF 的主要合成场所。生理条件下, TFF 的分布具有组织专一性, 具有黏膜保护和创 伤修复作用。TFF 在肿瘤组织中广泛表达, 在肿瘤的发生、发展、侵袭、转移过程中发挥着重要作用, 可能是癌 基因在对不同刺激做出反应时的共有递质。TFF 生物学功能存在一个复杂的调控过程, 单链 TFF 可通过膜受体而 激活相关信号通路, TFF 也可以通过与其它蛋白质的结合而协同发挥作用。TFF 在临床医学领域主要运用在稆膜 保护、黏膜损伤相关疾病的预防和治疗及肿瘤病理的诊断与干预等方面。TFF 的作用机制和功能行使相关信号通 路仍然是目前研究的重点, TFF 与 60 - 晶状体蛋白天然复合物的发现有助于对 TFF 的进一步认识和深入研究。

关键词: 三叶因子; 生理功能; 病理功能; 黏膜保护; 创伤修复; 肿瘤

中图分类号: Q959.5; Q51; R730.231 文献标识码: A 文章编号：0254-5853(2010)01-0017-010

\section{Trefoil Factor: from Laboratory to Clinic}

\author{
DU Ting-Yi ${ }^{1,2,3}$, ZHANG Yong ${ }^{1,3}$, ZHANG Yun ${ }^{1, *}$ \\ (1. Key Laboratory of Animal Models and Human Disease Mechanisms, Kunming Institute of Zoology, the Chinese Academy of Sciences, Kunming Yunnan \\ 650223, China; 2. Department of Clinical Laboratory Medicine, the First People's Hospital of Yunnan Province, Kunming , 650032, China; \\ 3. Graduate School of the Chinese Academy of Sciences, Beijing 100049, China)
}

\begin{abstract}
Trefoil factor (TFF) family is a group of peptides with one or several trefoil factor domains in their structure, which are highly conserved in evolution, and are characterized by heat and enzymatic digestion resistance. The mammalian TFFs have three members (TFF1-3), and the gastrointestinal tract and the airway system are major organs of their expression and secretion. At certain physiological conditions, with a tissue-specific distribution, TFF plays an important role in mucosal protection and wound healing. But in the malignant tissues, TFF is widely expressed, correlated strongly with the genesis, metastasis and invasion of tumor cells. These phenomena indicated that TFF may be a possible common mediator of oncogenic responses to different stimuli. The biological functions of TFF involve complex regulatory processes. Single chain TFF may activate cell membrane receptors and induce specific signaling transduction. On the other hand, TFF can form a complex with other proteins to exert its biological effects. In clinical medicine, TFF is primarily applied as drugs in the mucosal protection, in the prevention and the treatment of mucosal damage-related diseases and as pathological biomarkers of tumors. At present the first hand actions and the molecular mechanisms related to TFFs are still the major challenges in TFF research. Furthermore, the discovery of the naturally occurring complex of TFF and crystallins is highly valuable to the understanding of the biological functions and action mechanisms of TFF.
\end{abstract}

Key words: Trefoil factor; Physiological function; Pathological function; Mucosal protection; Wound restitution; Tumor

收稿日期：2009-12-07; 接受日期：2009-12-22

基金项目： 973 项目（2010CB529800); 国家基金委重点项目（30630014); 国家基金委面上项目（30870304); 中国科学院重要方向 项目(KSCX2-YW-R-088)

“通讯作者（Corresponding author）, E-mail: zhangy@mail.kiz.ac.cn

第一作者简介: 杜廷义(1966ï), 男, 博士研究生, 研究方向为肿瘤的分子生物学诊断研究, E-mail: timkally@hotmail.com 
$10^{4}$ )，具有耐热、耐酶消化的理化特性。TFF 在结 构上的共同点是具有一个或多个型似三叶草的三 叶因子结构域 (又称为 P 结构域), 这不仅是其理 化特性的结构基础（Wong et al,1999），也是其得 以命名的由来。

三叶因子结构域的最小共有序列为: C- $\mathrm{X}_{6-7}-\mathrm{R}-\mathrm{X}_{2}-\mathrm{C}-\mathrm{G}-\mathrm{X}_{8}-\mathrm{C}-\mathrm{X}_{4}-\mathrm{C}-\mathrm{C}-\mathrm{X}_{9}-\mathrm{W}-\mathrm{C}$ (Hoffmann \& Hauser, 1993), 其中的 $\mathrm{X}$ 代表任意氨基酸残基, 整个基序约 38 39 个氨基酸残基。序列中所含的 6
个半胱氨酸残基以 1-5，2-4，3-6 的配对方式形成 分子内二硫键, 在很大程度上决定了 $\mathrm{TFF}$ 分子结构 和稳定性(Thim, 1989)。20 世纪 90 年代开始, TFF 蛋白二级结构和空间结构得到了较为深入的研究 (Carr et al，1994; De et al, 1994; Polshakov et al, 1995), 其空间结构、理化特性、功能位点等表征特 性现已基本明确。图 1 为 TFF1 的单体结构示意图。

TFF 主要发现于脊椎动物, 如哺乳动物、人、 大猩猩、猴、猪、狼、猫、鼠等和两栖类动物。
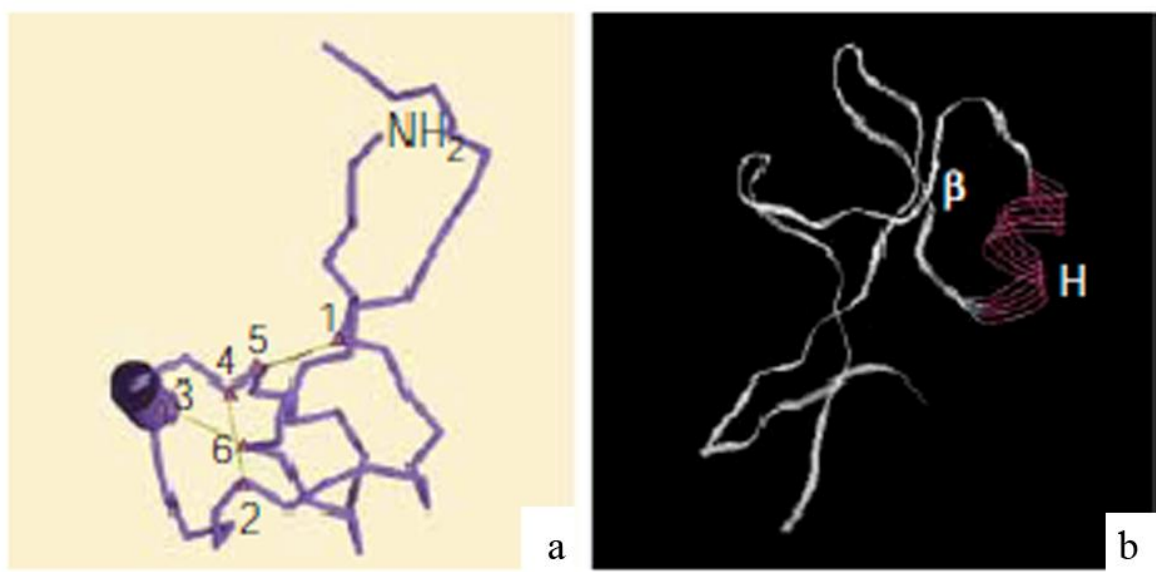

图 1 三叶因子单体结构示意图

Fig. 1 Trefoil factor structure

$\mathrm{a} ：$ TFF1 单体的正交图; b : 线状图。三叶因子结构域中，从氨基端开始的六个保守的半胱氨酸残基以 1ï 5、2ï 4、3ï 6 相互配 对的方式形成分子内二硫键，构成紧凑的三环结构。二级结构特征为一个短螺旋 $(\mathrm{H})$ 与相邻的两个 6 折叠条带形成一个反向平行 6片层(b)(引自 Taupin \& Podolsky, 2003)。

Orthogonal (a) and ribbon (b) diagrams of monomeric TFF1. The conserved cysteines of the trefoil domain are numbered from the amino terminus, and form three disulphide bonds in the 1-5,2-4 and 3 - 6 configuration. This produces a compact three-loop structure. The secondary structure is limited to a short helix $(\mathrm{H})$, adjacent to two 6 strands that form an antiparallel $\mathrm{b}$ sheet (6) (cited from Taupin \& Podolsky, 2003).

在进化上, TFF 成员的序列结构具有高度的保守性, 同源类似蛋白在三叶因子结构域内的保守度高于 其他区域, 高度保守的氨基酸残基位点可能为结构 域中重要的结构和功能所必需 (Thim \& May, 2005)。

TFF 在脊椎动物中广泛的物种分布及进化保守 性提示其可能是一类具有重要生理病理功能的蛋 白质, 针对 TFF 所进行的长期而广泛的深入研究积 累了大量的成果。本文结合两栖动物三叶因子研究 的新进展, 重点介绍 TFF 生理病理功能的研究概况 及其在临床医学中的应用发展。

\section{TFF 的表达与分布}

\subsection{TFF 在两栖类动物的表达}

两栖类动物皮肤是分离生物活性多肽的重要 来源。Hoffmann (1988) 从非洲爪蟾 (Xenopus laevis)
皮肤中克隆了一个含有四个三叶因子结构域的前 体蛋白。随后的研究证明, 这个前体蛋白是以一个 含有四个三叶因子结构域的高分子量的高度糖基 化蛋白的形式存在于蛙皮肤, 该蛋白随即被命名为 蛙皮黏液素 A1（FIM-A1）（Hauser et al，1990）。 蛙皮黏液素家族的另一成员即含六个三叶因子结 构域的 FIM-C1（Hauser \& Hoffmann，1992a）。同 期, 一个来自蛙消化道的只含一个三叶因子结构域 的热稳定蛋白（Gmachl et al，1990）随即被发现。 除了这些大分子量的皮肤蛋白外，在蛙皮还存在含 二个三叶因子结构域的小分子多肽 xP2 (Hauser et al, 1992b)。在非洲爪蟾胃中, 研究发现了含一个 三叶因子结构域的 $\mathrm{xP} 1$, 以及含四个三叶因子结构 域的 $\mathrm{xP} 4$ 。它们的表达方式非常类似于人 TFF1 和 TFF2 (Hauser F \& Hoffmann, 1991)。较近的研究 还发现，在大蹼铃蟾（Bombina maxima）皮肤分泌 
物 中存在含两个三叶因子结构域的 Bm-TFF2(Zhang et al, 2005), 以及含三个三叶因子 结构域的 TFF 与 62 -晶状体蛋白的天然复合物 (Liu et al, 2008a)。

蛙类动物含三叶因子结构域的多肽蛋白的功 能并不是很清楚的; 但由于存在古在青蛙皮肤中的 每一种蛋白多肽都将在哺乳动物中找到ò这一现 象, 同时由于它们的表达方式类似于哺乳动物三叶 因子多肽蛋白, 可以推测蛙类动物三叶因子多肽蛋 白的功能可能与哺乳动物 TFF 相类似。因此, 对脊 椎动物的研究可以为哺乳动物 TFF 的科研工作提 供新的工具、线索和更广泛的可行性(Qian et al, 2008a)。

\subsection{TFF 在哺乳动物体内的组织表达与分布}

1.2.1 TFF 在正常组织中的表达分布 哺乳动物的 TFF 有三个成员: TFF1 (乳腺癌相关肽, $\mathrm{pS} 2$ ) 、 TFF2 (解痉多肽, SP) 和 TFF3 (肠三叶因子, ITF)。 TFF 在整个消化系统中 (胃肠道、肝胆、胰腺等) 均有表达分布。胃肠道是 TFF 的主要合成场所, TFF 的分布具有较强组织专一性, TFF1 和 TFF2 在胃部

(Kjellev et al, 2006; Kjellev et al, 2007), TFF3 则 主要在大肠和小肠呈高表达 (Baus-Loncar et al, 2005 ); 而在发生溃疡等病理改变时, 这种组织专 一性消失（Rio et al，1991; Theisinger et al, 1991; Wright et al, 1993)。生理条件下, TFF 由具有分泌 功能的杯状细胞、黏膜细胞、腺细胞等合成并分泌 进入管腔, 可以与黏液蛋白 (MUC) 共同发生作用。 研究发现, 在胃肠道内, TFF 与 MUC 之间存在着 各自独特的共表达模式, 如 TFF1 和 MUC5AC

(Hoffmann et al, 2001)、TFF2 与 MUC6 (Matsuoka et al, 1999)、TFF3 与 MUC2 (Longman et al, 2002) 等。一般情况下, 这种共表达方式在不同物种间表 现一致, 这从另一角度反应了 TFF 在进化上的保守 性。

除消化系统外, 大量的文献表明 TFF 在其他组 织的黏膜上皮细胞中也有表达, 如眼睛的角膜、结 膜和泪腺导管、唾液腺、呼吸道黏膜、前列腺、生 殖器官、淋巴组织、下丘脑、垂体、心肌和骨骼肌 等等。TFF 在肠道外组织的表达虽然广泛, 但在不 同组织之间表达水平变异较大。这种多种器官组织 均分别有不同 TFF 成员、不同量的表达分布情况, 提示出 TFF 作用的多样性、广泛性和复杂性, 比如 在人下丘脑和垂体组织中分离到的 TFF3 就具有神
经肽样的作用（Probst et al,1996）。

近期的研究报道, 在哺乳期妇女的乳液中存在 高浓度的 TFF1 和 TFF3 (Vestergaard et al, 2008), 在健康人群的尿液中检出 TFF1(Chutipongtanate et al, 2005)。在继对血液中的 TFF 开展研究之后, TFF 在体液中的分布和功能引起了进一步的关注。

1.2.2 TFF 在病理情况下的表达 TFF 在病理情况 下的表达研究主要集中在病变时 TFF 的异位表达、 异常量表达, 以及实体肿瘤的异常表达和疾病模型 研究等几个方面。

在慢性肠胃炎、消化性溃疡、增生性息肉、肠 化生、胃肠道肿瘤等几乎各种与胃肠道黏膜组织有 关的病变及胰腺、肝胆系统的病变中都可发现 TFF 的异位表达和/或异常量表达。针对疾病模型的研究 结果与实际病理条件下的改变基本一致。TFF 在人 类病理状态和动物疾病模型中的表达简况参见表 1 。

在研究胃肠道发生病理改变, TFF 异常表达时, 溃疡相关细胞系 (UCAL) 的发现 (Wright et al, 1990a）引起了广泛的关注。UCAL 是紧邻溃疡病变 区域、由肠隐窝基底形成的一个腺样结构, 该细胞 系的细胞似乎直接来源于干细胞区 (Ahnen et al, 1994; Patel et al, 1994)。UCAL 表达生长因子 （TGF、EGF）及三种已知的 hTFF 蛋白 (Hauser et al, 1993), 其发育与布鲁纳氏腺的发育有些相似, hTFF 蛋白的表达模式也基本相同（Ahnen et al, 1994)。同时有研究报道: 在与结肠损伤相关的增 生性息肉中, 发现了一个新的细胞系, 其表达 hTFF 蛋白的模式类似于 UCAL (Hanby et al, 1993), 类 似的表达现象在非胃肠道组织中也有发现 (Wright et al，1990b)。UCAL 及其与之相似的细胞系的发 现, 强烈地体现出创伤修复是一个受到精细调控 的、多种因素参与的生物过程, 这一过程的精密性、 复杂性尚需更加深入的研究加以揭示。

TFF1 首先是在乳腺癌细胞系 MCF-7 中被发现 的, 并随之进行了基因全序列克隆 (Masiakowski et al, 1982; Jakowlew et al, 1984）。此后, 大量的研 究发现了 TFF 家族蛋白在多种实体瘤中的异常表 达。已报道的实体瘤分布于除运动系统和循环系统 以外的身体各个系统, 如: 乳腺癌、食管癌、胃癌、 胰腺癌、胆管癌、肺癌、前列腺癌、肾癌、妇科癌 症、神经内分泌肿瘤等。实体瘤组织中的异常表达 大多为 TFF1 和 TFF3, 但在胰腺癌和前列腺癌病 
例中，也有 TFF2 表达的研究报道 Collier et al,1995；Vestergaard et al, 2006）。

表 1 TFF 在人类病理状态和动物疾病模型中的表达简况

Tab. 1 Expression of TFF in human pathology and animal models of human disease

\begin{tabular}{lc}
\hline \multicolumn{1}{c}{ 病理情况 / 动物模型 Pathology / Model } & TFF 表达情况 TFF \\
\hline Barretô esophagus & $1-3(\hat{\mathrm{y}})$ \\
GERD & $3(\hat{\mathrm{y}})$ \\
Peptic ulcers & $1,2(\hat{\mathrm{y}})$ \\
IBD & $1-3(\hat{\mathrm{y}})$ \\
Chronic diverticulitis & $1,2(\hat{\mathrm{y}})$ \\
Cholecystitis & $1,2(\hat{\mathrm{y}})$ \\
Biliary disease & $1-3(\hat{\mathrm{y}})$ \\
Pancreatitis & $1(\hat{\mathrm{y}})$ \\
Numerous cancers & $1-3(\hat{\mathrm{y}} \dot{z})$ \\
Gastric ulcer (rat) & $2,3(\hat{\mathrm{y}})$ \\
Acetic acid-induced colitis (rat) & 1,3 \\
Methotrexate-induced enteritis (mice) & $3(\hat{\mathrm{y} Z ́})$ \\
Methotrexate + radiation (mice) & $3(\hat{\mathrm{z}})$ \\
DSS-induced colitis (mice + rats) & $3(\hat{\mathrm{y}})$ \\
DNBS-induced colitis & $2(\hat{\mathrm{y}})$ \\
T cell adoptive transfer colitis (mice) & $3(\hat{\mathrm{y}})$ \\
Asthma (mice) & $1,2(\hat{\mathrm{y}})$ \\
\hline
\end{tabular}

(Ŷ): 表达增高; (Ź): 表达减少; GERD:胃 $\mathrm{i}$ 食管反流性疾病; IBD: 炎性肠病; DSS: 葡聚糖硫酸钠; DNBS: 二硝基苯磺酸(引自 Kjellev, 2009)。 Note: (̂ิ): increased expression; (Ź): decreased expression; GERD: gastro-esophageal reflux disease; IBD: inflammatory bowel disease; DSS: dextrane sulphate sodium; DNBS: dinitrobenzenesulphonic acid (cited from Kjellev, 2009).

综合大量文献: TFF 在恶性组织中的广泛表达, 表明其在组织的恶变过程中发挥着一定的作用。但 在不同的肿瘤组织中，在肿瘤不同的发生、发展、 转移、恶化阶段, TFF 异常表达方式不一, 在某些 病理情况下表现为进行性上调; 另外一些情况又表 现为下调 (May \& Westley, 1997; Regalo et al, 2005）; 生理作用也表现不同, 甚至是相反, 比如 TFF1 在胃癌中发挥肿瘤抑制因子的作用, 而在结 肠中则发挥着致癌因子的作用 (Lefebvre et al, 1996; Rodrigues et al，2006）。TFF 在肿瘤病理中 的这种表达和作用模式的多样性与其功能密切相 关, 同时也进一步证明 TFF 的生理作用存在一个复 杂的调控过程。TFF 在动物体内的生理作用和分子 生物学机制、细胞生物学机制问题, 尽管已经取得 了广泛而深入的研究成果, 但至今仍未得到较为彻 底的解答。

\section{TFF 的生理功能和病理作用}

在 TFF 已知的生物学功能当中, 与临床医学关 系最为紧密, 受到临床医学更多关注的是黏膜保护 与创伤修复及肿瘤病理两个大的方面; 而 TFF 的其 他功能性问题, 相对来说与基础医学的关系更为密 切。

\subsection{TFF 的黏膜保护和愈合促进作用}

TFF保护胃肠道黏膜上皮, 抵御外源因素诱导 损伤的作用已得到多方面的研究证实。

Babyatsky研究小组通过胃管灌注乙醇、皮下注 射非固醇类抗炎药物吲哚美辛（indomethacin）两 种方式建立大鼠胃损伤模型。研究证明hTFF2、 rTFF3对两种方式诱导的大鼠胃损伤均有明显的保 护作用（Babyatsky et al, 1996)。其他研究也证明了 $\mathrm{TFF} 1$ 的类似作用。

Dignass及其合作者采用受伤的单层融合细胞, 建立了离体的上皮细胞恢复模型, 研究发现: 当加 入TFF2或TFF3时, 上皮细胞运动迁移到伤口部位 的速度增加 $3 \sim 6$ 倍; 当 TFF多肽蛋白与黏膜糖蛋白 
同时加入, 上皮细胞运动速度增加15倍 (Dignass et al, 1994)。

Playford 及其同事建立了小鼠转基因动物模 型, 该模型动物能在空肠线毛区域过量表达人 TFF1。结果显示由吲哚美辛诱导的胃部病变程度明 显低于对照动物, 因而从转基因动物水平上确认了 $\mathrm{TFF}$ 多肽蛋白在黏膜损伤修复过程中的功能 (Playford et al, 1996)。

Mashimo et al（1996）通过基因打靶技术，分 别敲除小鼠 TFF2、TFF3 基因。缺失 TFF3 基因的 小鼠在口服硫酸葡聚糖后, 受损的黏膜不能恢复, 动物常常死于大面积肠炎; 而 TFF1 和 TFF2 的表 达是正常的。试验结果显示 TFF1 和 TFF2 不能弥 补 TFF3 的缺失。

进一步的动物研究结果显示, 在吲哚美辛及精 神紧张诱发的胃创伤活体动物中, 在受损前 30 $\min$ 及受损同时皮下注射 $25 \mu \mathrm{g} /(\mathrm{kg} \cdot \mathrm{h})$ 小剂量的 hTFF2 均有效 (Playford et al, 1995; Konturek et al, 1997)。而在受损部位施用重组 TFF3, 能够有效地 产生保护作用和促进愈合作用的试验结果是 TFF3 参与黏膜保护和黏膜修复功能的最直接的证据 (Babyatsky et al, 1996; Chinery \& Playford, 1995; Mashimo et al, 1996)。

Hoffmann et al (2001) 和 Kjellev (2009) 的 研究总结表明: TFF 对黏膜的保护作用的机制在于 稳定黏液层及刺激上皮细胞在伤口修复过程中的 恢复愈合, 包括: (1) TFF 多肽蛋白与黏膜糖蛋白协 同形成稳定的凝胶状复合物, 该复合物可抑制胃肠 蛋白酶的作用和抵抗机械应力对胃肠道黏膜的损 伤。(2) TFF 多肽蛋白作为致动原, 可诱导小肠及呼 吸系统上皮细胞的迁移, 促进秥膜创伤部位的愈 合。黏膜糖蛋白可增强 TFF 的这种作用。(3) TFF 多肽蛋白具有生长因子或协调其它生长因子的作 用, TFF2 还通过刺激胃肠道细胞的增殖和抑制胃 酸的分泌来促进上皮细胞的修复和控制局部炎症。

(4) TFF 具有抑制细胞调亡, 保护黏膜细胞的作用。

(5) TFF 对黏膜保护功能和促进创伤愈合功能是与 其它因素 (如免疫球蛋白、细胞因子及生长因子等 等）通过一系列的信号传导途径共同作用完成的。

\subsection{TFF 在肿瘤病理过程中的作用}

在多种实体瘤组织中存在 TFF 的异常表达。

TFF 在肿瘤发生、发展、侵袭转移中的作用备受关 注, 并得到以下深入的研究:
胃癌す す TFF1 对胃癌的发生具有抑制作用。 对胃癌的研究发现部分病例存在编码 TFF 的染色 体 21q22.3 区域的丢失现象，而 TFF1 表达水平在 胃癌组织中较周围的正常组织明显下降。在此现象 提示下对小鼠模型进行的研究表明, TFF1 缺陷型 的小鼠会发生前幽门瘤, 其中近 $1 / 3$ 将恶化为上皮 内、黍膜内的癌症以及胃腺癌 (Lefebvre et al,1996)。 进一步的研究发现 TFF1 的缺失造成对胃黏膜损伤 修复的能力下降, 从而导致消化道的炎症反应出 现, 增加了胃癌发生的几率。Beckler et al (2003) 对原发性胃癌病例的研究发现: 与正常组相比较, 胃癌病理组织中的 TFF1-mRNA 和蛋白质显著减 少, 但未见编码基因的突变。随后的研究报告也支 持这一结论（Ren et al, 2005; Moss et al, 2008）。 而 Kim et al（2008）的研究结果显示：与原发性胃 癌相比较, 转移性胃癌的 TFF1 蛋白表达量明显增 加。TFF3 在胃癌病例中的异常表达相对 TFF1 而言, 表现更为复杂, 在同一组病例中, 有的表现增加而 有的减少（Kirikoshi \& Katoh, 2002）。

肝胆癌症す ठ Khoury et al (2005) 对肝细胞 癌组织中 TFF3 表达情况的研究结果显示: TFF3 的 表达水平与肿瘤分级之间存在较强的相关性, 而与 存活率之间没有相关性。Thuwajit et al (2007) 对 病理组织和肿瘤细胞系进行的研究提示: TFF1 与 MUC-5AC 在肝内胆管癌组织中表现出较高水平的 共表达现象, TFF1 可能对癌症的侵袭具有促进作 用。Haruhiko et al (2007) 等对病例样本和动物模 型的研究显示: 在 mRNA 和蛋白质表达水平上, TFF3 均有升高, 动物模型与病例样本的主要差别 在于 14 例小鼠模型中有 13 例表现出较高表达水 平，而 20 例病例样本仅有 8 例表现出升高。

结肠癌す 更为值得关注的是 Yio et al (2005) 和 Babyatsky et al (2008) 研究小组应用 siRNA 构 建技术, 结合多种研究方法, 先后对体外细胞株, 疾病动物模型和病例样本进行了 TFF 与肿瘤侵袭 性生物行为的关系研究, 结果显示 TFF3 与肿瘤转 移相关。这为 TFF 对肿瘤转移具有促进作用提供了 比较完整的试验证据。

乳腺癌す 乳腺癌是最早进行 TFF 在癌症病 理中的作用研究的样本之一, TFF1 的表达与否可 以预示内分泌辅助治疗的疗效也早已得到肯定。近 期的研究主要关注 TFF 在乳腺癌侵袭转移过程中 的作用。May et al (2004) 的研究结果显示: TFF2 
在正常细胞和恶性肿瘤上皮细胞中均有表达, 并且 可以刺激癌细胞的迁移。Smid et al (2006) 发现 在乳腺癌细胞转移行为中, 与身体其它部位的转移 相比较, 癌细胞的骨转移具有独特的基因表达特 征, 其中包括 TFF1、TFF3（TFF1 与癌细胞骨转移 之间的相关性更为明显）, 进而提出特定器官转移 的特殊基因表达的假设。

TFF 在肿瘤病理过程中的作用一直备受临床医 学关注。尽管近期的研究在作用机制、信号通路等 方面取得了一些成果, 也有新的假设提出; 但由于 三叶因子的分子作用方式, 潜在的受体及分子作用 机制一直未能得到阐明, 对于 TFF 与肿瘤发生、发 展之间关系的理解仍然建立在已知的 TFF 的生理 功能和对试验结果的推理的基础上。

$\mathrm{TFF}$ 的生物学功能复杂而多样, 参与了动物体 内的多种生理反应和病理反应。大量研究结果表 明: TFF 参与了黏膜保护 (Chinery \& Playford, 1995; Playford et al, 1996)、创伤修复 (Goke et al, 2001)、 细胞迁移 (Kato et al,1999; Prest et al, 2002; Graness et al,2002)、调亡调控 (Kinoshita et al, 2000; Taupin et al,2000）、肿瘤病理（Calnan et al，1999; Emami et $\mathrm{al}, 2004$ ) 、细胞分化 ( Taupin et al,2001; Bossenmeyer-pourie et al, 2002) 、血管生成 (Rodrigues et al，2003）、免疫调节（Cook et al, 1999）等重要的生物学过程。对研究结果的分析和 更多的实验研究进一步说明:

(1) TFF 缺失与炎症反应的作用。 TFF1 缺失 将导致肠道的炎症性紊乱, 炎症侵润进一步使肠道 黏膜的完整性受到不可逆的破坏, 肠道组织发生转 化, 转化的组织可能进而恶化为肿瘤组织。

（2）TFF 可能是潜在的肿瘤发生因子。这一 潜在作用主要是与 TFF 对肿瘤细胞所具有的抗调 亡活性、促细胞迁移活性相联系的。同时, 实体瘤 的发生和肿瘤的转移都依赖于新生血管的形成, $\mathrm{TFF}$ 的血管生成因子活性可能在肿瘤发生、转移过 程中发挥作用。

（3） TFF 对肿瘤细胞株具有抑制生长作用。 TFF 的缺失将可能直接影响机体对肿瘤细胞生长的 控制。TFF 抑制肿瘤细胞生长的作用机制, 已知的 有以下主要途径: (1) 降低 MAPK/ERK 的磷酸化; (2) 增加细胞周期激酶抑制剂 p16INK4 和 $\mathrm{p} 21 \mathrm{CIP} 1 / \mathrm{Waf} 1$ 水平; (3) 抑制 cyclinD1/cdk4 和 cyclinE/cdk2 复合物的活性。
虽然作用机制仍未明确和试验研究结果存在 不一致, 甚至矛盾的现象; 但 TFF 与肿瘤的发生、 发展、侵袭转移密切相关已是不争的事实。肿瘤是 一种多诱因, 多基因变异产生的因细胞分化、细胞 增殖过程调控失衡所造成的病理变化, 很难用单一 因素去完全解答这个复杂的病理变化过程。TFF 既 是肿瘤抑制因子，又是潜在的肿瘤发生因子，这种 作用的两面性长时间以来困惑了对 TFF 与肿瘤关 系的研究。曾经假设 TFF 编码基因本质上可能是一 个癌基因; 但这一假设难以对 TFF 作用的两面性做 出较为圆满的解答。在肿瘤病理过程中, TFF 可能 扮演的是癌基因在对不同刺激做出反应时的共有 递质 (common mediator) 的角色 (Perry et al,2007); 如果这一推测被证实, 那么, TFF 在肿瘤的发生、 发展、预防治疗中的作用, 就将会有一个新的认识 角度和应用可能。

\section{TFF 研究在临床医学中的应用}

TFF 在临床医学领域的应用, 必然是建立在广 泛而深入的实验室研究基础上的。TFF 发现以来, 其在临床医学实践中可能具有的作用一直是研究 者们所关注的重点之一。经过长期的努力, 在成熟、 可靠的前提下，目前能进入临床实践的仅为 $\tilde{n}$ 基因 重组 TFF30̀。

“基因重组 TFF3”已被批准为国家一类新药, 其治疗适应症为修复黏膜、预防与治疗胃肠道溃疡 及炎症，也可用于对放疗、化疗造成的黏膜损伤的 辅助支持治疗。“基因重组 TFF3” 目前可使用的 为口服剂和注射剂两种剂型。随着更多的临床实践 评估报道的发表, 以及临床疗效的证实及使用范围 的推广，相信新的剂型和适用范围将会得到更新。

“基因重组 TFF3” 还具有使用在化妆品和营养保 健品的潜在价值。

黏膜保护不会是 TFF3 唯一的临床使用范围。 与黏膜损伤、创伤修复相关的疾患如结石症、疤痕 修复等都可能是 TFF3 发挥作用的领域。甚至可能 还会有新的领域, 比如 Chutipongtanate et al(2005) 对尿液中检出的 TFF1 进行的研究发现, TFF1 具有 抑制草酸钙结晶性质的尿结石在尿路中滞留生长 的功能; 而 Vestergaard（2008）研究团队的研究报 道提示了基于黏膜保护作用的, TFF 在新生儿医学 实践上的应用可能。

Vestergaard et al （2008）对新生儿母乳中的 
TFF 含量进行连续观测发现: 在哺乳母亲的产后第 一周的乳汁中含有较高水平的 TFF1 和高水平的 TFF3, 并在两周内快速下降, 产后 $15 \mathrm{~d}$ 降至正常 个体水平并保持稳定。进一步的分析认为, 母乳中 的 TFF3 的低水平与新生儿坏死性小肠结肠炎的发 生风险相关。对上述现象之间关系的进一步研究将 有助于改进配方奶成分以降低早产新生儿坏死性 小肠结肠炎的发病风险。

相对于黏膜保护功能的开发利用, TFF 在肿瘤 医学中的应用尚处于探索阶段。TFF 在肿瘤治疗中 的应用开发不仅有待于对 TFF 在肿瘤病理过程中 作用机制的进一步研究和认识, 同时也有待于靶向 治疗、基因治疗技术的成熟和发展。尽管如此, 近 期的研究显示, 随着检测技术的进步和大量研究资 料的积累, 作为生物学标志物— $\mathrm{TFF}$ 在肿瘤的诊 断和鉴别诊断（Bosma et al,2002; Takano \& Yamada，2009）、疗效监测（Bignotti et al,2008）、 预后判断 (Yamachika, 2002) 等肿瘤诊断学实践 中的应用已为期不远。

\section{4 展 望}

作为一个较新的蛋白质家族, TFF 在 20 世纪 80 年代被发现, 90 年代后期得到系统的分类和命 名。通过近 20 年来的研究工作, 对 TFF 的认识已 经取得了大量的成果。基因敲除研究 (Lefebvre et al, 1996; Mashimo et al, 1996)清楚地表明：如果没 有 TFF 的正常表达, 上皮细胞修复再生的动态过程 就会被严重破坏。毫无疑问, 对胃肠道系统的正常 生理功能和对上皮细胞的修复再生过程, TFF 多肽 具有重要的作用。肿瘤病理学方面的研究成果明确 显示: TFF 广泛参与了细胞生物学过程, 影响着肿 瘤细胞的发生、发展, 参与了肿瘤细胞的侵袭和转 移。可以预期, 随着对 TFF 生物学功能认识的加速, 它将在临床医学领域得到越来越广泛的运用。

TFF 的临床应用取决于基础研究的进展。进一 步阐明 TFF 多肽分子的功能; 鉴定其受体或结合蛋 白; 彻底弄清其行使功能时的各级信号通路仍然是 目前 TFF 研究的重要问题。近期对两栖类 TFF 的 一些研究成果为今后的研究工作提供了有益的提 示。
非晶状体 6 כ 晶状体蛋白与 TFF 蛋白复合物 (non-lensb2-crystallin and trefoil factor complex, b2-CAT)为第一个从冷椎动物皮肤分泌物中分离得 到的全新的天然蛋白复合物, 其组成亚基分别与人 类黑色素瘤缺失蛋白 I 和 hTFF 高度同源 (Liu et al, 2008a)。 D2-CAT 具有诱导形成细胞膜孔道 (Liu et $\mathrm{al}, 2008 \mathrm{~b}$ ）, 细胞囊泡化形成和细胞内转运及杀伤 肿瘤细胞（He et al，2008 a ）、诱导兔胸动脉环持 续收缩（Liu et al，2008c）等多方面的生物学活性

(He et al, 2008b; Qian et al, 2008b)。62-CAT 在皮摩尔浓度水平对细胞具有促进迁移和修复的 作用, 而在毫摩尔水平则会导致黏壁细胞脱落, 并 诱导细胞调亡。进一步的细胞分子生物学试验发现 在毫摩尔水平时, 细胞以快速囊泡化的方式将 b2-CAT 转运至细胞核区域, 与 hTFF 高度同源的 60 亚基主要集中于胞内区域, 并进而导致细胞与有丝 分裂、细胞分化、细胞生长、组织再生与修复有密 切关系的相关基因表达谱的显著变化。6כ-CAT 在 研究中表现出的对细胞迁移、细胞凋亡的作用特征 与 TFF 非常相似。因此有理由认为对 b2-CAT 的深 入研究可以为 hTFF 的生物学功能研究提供有益的 参考。 $\mathrm{b}-\mathrm{CAT}$ 的研究结果提示 TFF 在体内作用的 复杂性, 即单链 TFF 可通过膜受体而激活相关信号 通路而发挥其生物学功能; 另一方面, TFF 也可以 通过与其它蛋白质的结合而协同发挥作用。这为揭 示久而未决的哺乳动物 TFF 分子作用机制提供了 一个新的方向, 也为对 TFF 功能实现方式的理解提 供了一个新的思路和分子模型。

综上所述, TFF 是一类在生物体内具有重要生 物学功能的调节因子, 主要行使机体维护和自稳态 维持机能。TFF 的多重和复杂生物学功能可由单链 $\mathrm{TFF}$ 直接发挥作用, 亦可通过 TFF 与其它蛋白质的 复合物而发挥作用。TFF 的生理病理功能与细胞增 殖分化、组织再生修复密切相关, 其作为抑癌基因 和/或癌基因的可能性有待进一步认识。结合脊椎 动物不同物种 TFF 的研究 (特别是新的生物学功能 和分子作用机制的发现, 具有广泛生物学活性的天 然 TFF 单体及其 TFF 复合物的纯化和研究) 将使 对 TFF 的认识更加深入细致。可以相信, TFF 在临 床医学领域更为广泛的应用前景已不再遥不可及。 


\section{参考文献:}

Ahnen DJ, Poulsom R, Stamp GWH, Elia G, Pike C, Jeffery R. 1994. The ulceration - associated cell lineage (UACL) reiterates the Brunnerô gland differentiation program but acquires the proliferative organization of the gastric gland [J]. J Pathol, 173(4): 317-326.

Babyatsky MW, deBeaumont M, Thim L, Podolsky DK. 1996. Oral trefoil peptides protect against ethanol-induced and indomethacin-induced gastric injury in rats [J]. Gastroenterology, 110(2): 489-497.

Babyatsky M, Lin J, Yio X, Chen A, Zhang J, Zheng Y, Twyan C, Bao X, Schwartz M, Thung S, Werther JL, Itzkowitz S. 2008. Trefoil factor-3 expression in human colon cancer liver metastasis [J]. Clin Exp Metastasis, 26(2): 143-151.

Baus-Loncar M, Kayademir T, Takaishi S, Wang T. 2005. Trefoil factor family 2 deficiency and immune response [J]. Cell Mol Life Sci, 62(24): 2947-2955

Beckler AD, Roche JK, Harper JC, Petroni G, Frierson HFJ, Moskaluk CA, EI-Rifai W, Powell SM. 2003. Decreased abundance of trefoil factor 1 transcript in the majority of gastric carcinomas [J]. Cancer, 98(10): 2184-2191.

Bignotti E, Ravaggi A, Tassi RA, Calza S, Rossi E, Falchetti M, Romani C, Bandiera E, Odicino FE, Pecorelli S, Santin AD. 2008. Trefoil factor 3: a novel serum marker identified by gene expression profiling in high-grade endometrial carcinomas [J].Bri J Cancer, 99(5): 768-773.

Bosma AJ, Weigelt B, Lambrechts AC, Verhagen OJHM, Pruntel R, Hart AAM, Rodenhuis S, vanâ Veer LJ. 2002. Detection of circulating breast tumor cells by differential expression of marker genes [J]. Clin Cancer Res, 8: 1871-1877.

Bossenmeyer-Pourie C, Kannan R, Ribieras S, Wendling C, Stoll I, Thim L, Tomasetto C, Rio MC. 2002. The trefoil factor 1 participates in gastrointestinal cell differentiation by delaying G1-S phase transition and reducing apoptosis [J]. $J$ Cell Biol, 157(5): 761-770.

Calnan DP, Westley BR, May FEB, Floyd DN, Marchbank T, Playford RJ. 1999. The trefoil peptide TFF1 inhibits the growth of the human gastric adenocarcinoma cell line AGS [J]. J Pathol, 188(3): 312-317.

Carr MD, Bauer CJ, Gradwell MJ, Feeney J. 1994. Solution structure of a trefoilï motif-containing cell growth factor, porcine spasmolytic polypeptide [J]. Proc Natl Acad Sci USA, 91(6): 2206-2210.

Chinery R, Playford RJ. 1995. Combined trefoil factor and epidermal growth-factor ia prophylactic against indomethacin-indeced gastric damage in the rat [J]. Clin Sci, 88(4): 401-403.

Chutipongtanate S, Nskagawa Y, Sritippayawan S, Pittayamateekul J, Parichatikanond P, Westley BR, May FEB, Malasit P, Thongboonkerd V. 2005. Identification of human urinary trefoil factor 1 as a novel calcium oxalate crystal growth inhibitor $[\mathrm{J}] . J$ Clin Invest, 115:(12):3613-3622.

Collier JD, Bennett MK, Bassendine MF, Lendrum R. 1995. Immunolocalization of $\mathrm{ps} 2$, a putative growth factor, in pancreatic carcinoma [J]. J Gastroenterol Hepatol, 10(4): 396-400.

Cook GA, Familari M, Thim L, Giraud AS. 1999. The trefoil peptides TFF2 and TFF3 are expressed in rat lymphoid tissues and participate in the immune response [J]. FEBS Lett, 456(1): 155-159.

De A, Brown DG, Gorman MA, Carr MD, Sanderson MR, Freemont PS. 1994. Crystal structure of a disulphide-linked árefoilômotif found in a large family of putative growth factors [J]. Proc Natl Acad Sci USA, 91(3): 1084-1088

Dignass A, Lynch-Devaney K, Kindon H, Thim L, Podolsky DK. 1994. Trefoil peptides promote epithelial migration through a transforming growth factor beta-independent pathway [J]. J Clin Invest, 94(1): 376-383.
Emami S, Rodrigues S, Rodrigue CM, Le Floch N, Rivat C, Attoub S, Bruyneel E, Gespach C. 2004. Trefoil factors family (TFF) peptides and cancer progression [J]. Peptides, 25(5): 885-898.

Gmachl M, Berger H, Thalhammer J, Kreil G. 1990. Dermal glands of Xenopus laevis contain a polypeptide with a highly repetitive amino acid sequence [J]. FEBS Lett, 260(1): 145-148.

Goke MN, Cook JR, Kunert KS, Fini ME, Gipson IK, Podolsky DK. 2001 Trefoil peptides promote restitution of wounded corneal epithelial cells [J]. Exp Cell Res, 264(2): 337-344.

Graness A, Chwieralski CE, Reinhold D, Thim L, Hoffmann W. 2002. Protein kinase $\mathrm{C}$ and ERK activation are required for TFF-peptides-stimulated bronchial epithelial cell migration and tumor necrosis factorï a -induced interleukin-6 (IL-6) and IL-8 secretion [J].Biol Chem, 277(21): 18440-18446.

Hanby AM, Poulsom R, Singh S, Jankowski J, Hopwood D, Elia G. 1993. Hyperplastic polyps: a cell lineage which both synthesizes and secretes trefoil peptides and has phenotypic similarity with the ulcer associated cell lineage [J]. Am J Pathol, 142(3): 663-668.

Haruhiko O, Makoto TK, Tan DF, Kyoko F, Jun I, Masatoshi M, Hui AM, Masahiko T, Hiroki N. 2005. Frequent trefoil factor 3 (TFF3) overexpression and promoter hypomethylation in mouse and human hepatocellular carcinomas [J]. Int J Oncol, 26(2): 369-377.

Hauser F, Gertzen EM, Hoffmann W. 1990. Expression of spasmolysin (FIM-A. 1): an integumentary mucin from Xenopus laevis [J]. Exp Cell Res, 189(2): 157-162

Hauser F, Hoffmann W. 1991. xP1 and xP4, P-domain peptides expressed in Xenopus laevis stomach mucosa [J]. J Biol Chem, 266(31): 21306-21309.

Hauser F, Hoffmann W. 1992a. P-domains as shuffled cysteine-rich modules in integumentary mucin C. 1 (FIM-C.1) from Xenopus laevis. Polydispersity and genetic polymorphism [J]. J Biol Chem, 267(34): 24620-24624.

Hauser F, Poulsom R, Chinery R, Rogers LA, Hanby AM, Wright NA. 1993 hP1.B, a human P-domain peptide homologous with rat intestinal trefoil factor, is expressed also in the ulcer-associated cell lineage and the uterus [J]. Proc Natl Acad Sci USA, 90(15): 6961-6965.

Hauser F, Roeben C, Hoffmann W. 1992b. xP2, a new member of the $\mathrm{P}$-domain peptide family of potential growth factors, is synthesized in Xenopus laevis skin [J]. J Biol Chem, 267(20): 14451-14455.

He YY, Liu SB, Qian JQ, Lee WH, Zhang Y. 2008a. Mechanism of bב-CAT cell nuclear transportation and selectively killing of tumor cells [J]. Zool Res, 29(4): 386-398. [何英英, 刘树柏, 钱金桥, 李文辉, 张 云. 2008a. 非晶状体 60-晶状体蛋白与三叶因子蛋白复合物的细胞核转 运及其选择性杀伤肿瘤细胞机制的研究. 动物学研究, 29(4): 386-398.]

He YY, Liu SB, Lee WH, Zhang Y. 2008b. Melanoma cell growth inhibition by bुCAT, which is a novel non-lens betagamma-crystallin and trefoil factor complex from frog Bombina maxima skin [J]. Toxicon, 52(2): 341-347.

Hoffmann W. 1988. A new repetitive protein from Xenopus laevis skin highly homologous to pancreatic spasmolytic polypeptide [J]. J Biol Chem, 263(16): 7686-7690.

Hoffmann W, Hauser F. 1993. The P-domain or trefoil motif: a role in renewal and pathology of mucous epithelia ? [J]. Trends Biochem Sci, 18(7): 239-243.

Hoffmann W, Jagla W, Wiede A. 2001. Molecular medicine of TFF-peptides: from gut to brain [J]. Histol Histopathol, 16(1): 319-334. 
Jakowlew SB, Breathnach R, Jeltsch JM, Masiakowski P, Chambon P. 1984. Sequence of the pS2 mRNA induced by estrogen in the human breast cancer cell line MCF-7 [J]. Nucleic Acids Res, 12(6): 2861-2878.

Kato K, Chen MC, Nguyen M, Lehmann FS, Podolsky DK, Soll AH. 1999. Effects of growth factors and trefoil peptides on migration and replication in primary oxyntic cultures [J].Am J Physiol, 276(5Pt1): G1105-1116.

Khoury T, Chadha K, Javle M, Donohue K, Levea C, Iyer R, Okada H, Nagase H, Tan D. 2005. Expression of intestinal trefoil factor (TFF-3) in hepatocellular carcinoma $[\mathrm{J}]$. Int $J$ Gastrointest Cancer, 35(3): 171-177.

Kim JH, Kim MA, lee HS, Kim WH. 2008. Comparative analysis of protein expressions in primary and metastatic gastric carcinomas [J]. Hum Pathol, 40(3): 314-322.

Kinoshita K, Taupin DR, Itoh H, Podolsky DK. 2000. Distinct pathways of cell migration and antiapoptotic response to epithelial injury: structure-function analysis of human intestinal trefoil factor [J]. $\mathrm{Mol}$ Cell Biol, 20(13): 4680-4690

Kirikoshi H, Katoh M. 2002. Expression of TFF1, TFF2 and TFF3 in gastric cancer [J]. Int J Oncol, 21(5): 655-659.

Kjellev S. 2009. The trefoil factor family - small peptides with multiple functionalities [J]. Cell Mol Life Sci, 66(8): 1350-1369.

Kjellev S, Nexo E, Thim L, Poulsen SS. 2006. Systemically administered trefoil factors are secreted into the gastric lumen and increase the viscosity of gastric contents [J]. Br J Pharmacol, 149(1): 92-99.

Kjellev S, Thim L, Pyke C, Poulsen SS. 2007. Cellular localization, binding sites, and pharmacologic effects of TFF3 in experimental colitis in mice [J]. Dig Dis Sci, 52(4): 1050-1059.

Konturek PC, Brzozowski T, Konturek SJ, Elia G., Wright N, Sliwowski Z. 1997. Role of spasmolytic polypeptide in healing of stress-induced gastric lesions in rats [J]. Regul Peptides, 68(1): 71-79.

Lefebvre O, Chenard MP, Masson R, Linares J, Dierich A, Lemeur M, Wendling C, Tomasetto C, Chambon P, Rio MC. 1996. Gastric mucosa abnormalities and tumorigenesis in mice lacking the $\mathrm{pS} 2$ trefoil protein [J]. Science, 274(5285): 259-262.

Liu SB, He YY, Zhang Y, Lee WH, Qian JQ, Lai R, Jin Y. 2008a. A novel non-lens betagamma-crystallin and trefoil factor complex from amphibian skin and its functional implications [J]. PLoS ONE, 3(3): e1770.

Liu SB, He YY, Qian JQ, Zhang Y. 2008b. Electron microscopic analysis of bJ Res, 29(5): 503-510. [刘树柏, 何英英, 钱金桥, 张 云. 2008b. 电镜 分析非晶状体 60 晶状体蛋白与三叶因子蛋白复合物在人红细胞膜 上的孔道形成效应. 动物学研究, 29(5): 503-510.]

Liu SB, He YY, Qian JQ, Zhang Y. 2008c. Sustained contraction of isolated rabbit thoracic aortic rings in endothelial-dependent manner induced by bJ FAT [J]. Zool Res, 29(5): 493-502. [刘树柏，何英英，钱金桥，张 云. 2008c. 非晶状体 60 晶状体蛋白与三叶因子蛋白复合物诱导兔 胸主动脉环内皮依赖的持续收缩效应. 动物学研究, 29(5): 493-502.]

Longman RJ, Douthwaite J, Sylvester PA, Poulson,R, Corfield AP, Thomas MG, Wright NA. 2000. Coordinated localization of mucins and trefoil peptides in the ulcer associated cell lineage and the gastrointestinal mucosa [J]. Gut, 47(6): 792-800.

Mashimo H, Wu DC, Podolsky DK, Fishman MC. 1996. Impaired defense of intestinal mucosa in mice lacking intestinal trefoil factor [J]. Science, 274(5285): 262-265

Masiakowski P, Breathnach R, Bloch J, Gannon F, Krust A, Chambon P. 1982. Cloning of cDNA sequences of hormone-regulated genes from the MCF-7 human breast cancer cell line [J].Nucleic Acids Res, 10(24): 7895-7903
Matsuoka Y, Pascall JC, Brown KD. 1999. Quantitative analysis reveals differential expression of mucin (MUC2) and intestinal trefoil factor mRNAs along the longitudinal axis of rat intestine [J]. Biochim Biophys Acta, 1489(2-3): 336 -344.

May FE, Semple JI, Prest SJ, Westley BR. 2004. Expression and motogenic activity of TFF2 in human breast cancer cells [J]. Peptides, 25(5): 865-872.

May FE, Westley BR. 1997. Trefoil proteins: their role in normal and malignant cells [J]. Pathol, 183(1): 4-7

Moss SF, Lee JW, Sabo E, Rubin AK, Rommel J, Westley BR, May FE, Gao J, Meitner PA, Tavares R, Resnick MB. 2008. Decreased expression of gastrokine 1 and the trefoil factor interacting protein TFIZ1/GKN2 in gastric cancer: influence of tumor histology and relationship to prognosis [J]. Clin Cancer Res, 14(13): 4161-4167.

Patel K, Hanby AM, Ahnen DJ, Playford RJ, Wright NA. 1994. The kinetic organization of the ulcer-associated cell lineage (UACL): delineation of a novel putative stem-cell region [J]. Epithel Cell Biol 3(4): 156-160

Perry JK, Kannan N, Grandison PM, Mitchell MD, Lobie PE. 2007. Are trefoil factors oncogenic? [J]. Trends Endocrinol Metab, 19(2): 74-81.

Playford RJ, Marchbank T, Chinery R, Evison R, Pignatelli M, Boulton RA. 1995. Human spasmolytic polypeptide is a cytoprotective agent that stimulates cell migration [J]. Gastroenterology, 108(1): 108-116.

Playford RJ, Marchbank T, Goodlad RA, Chinery RA, Poulsom R, Hanby AM. 1996. Trangenic mice that overexpress the human trefoil peptides pS2 have an increased resistance to intestinal damage [J]. Proc Natl Acad Sci USA, 93(5): 2137-2142.

Polshakov VI, Frenkiel TA, Westley B, Chadwick M, May F, Carr MD, Feeney J. 1995. NMR-based structural studies of the pNR-2/pS2 single-domain trefoil peptideï similarities to porcine spasmolytic peptide and evidence for a monomeric structure [J]. Eur J Biochem, 233(3): 847-855.

Prest SJ, May FEB, Westly BR. 2002. The estrogen-regulated protein, TFF1, stimulates migration of human breast cancer cells [J]. FASEB, 16(6): 592-594.

Probst JC, Zetzsche T, Weber M, Theilemann P, Skutella T, Landgraf R, Jirikowski GF. 1996. Human intestinal trefoil factor is expressed in human hypothalamus and pituitary: evidence for a novel neuropeptide [J]. FASEB, 10(13): 1518-1523.

Qian JQ, Liu SB, He YY, Lee WH, Zhang Y. 2008a. Acute toxicity of bכ CAT, a naturally existing non-lens bכ-crystallin and trefoil factor complex from frog Bombina maxima skin secretions [J]. Toxicon, 52(1): 22-31.

Qian JQ, Liu SB, He YY, Lee WH, Zhang Y.2008b. 6Jf̧CAT, a non-lens

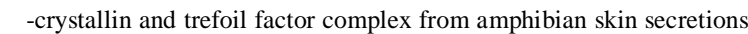
caused endothelium-dependent myocardial depression in isolated rabbit hearts [J]. Toxcin, 52(2): 285-292.

Regalo G, Wright NA, Machado JC. 2005. Trefoil factors: from ulceration to neoplasia [J]. Cell Mol Life Sci, 62(24): 2910-2915.

Ren JL, Luo JY, Lu YP, Wang L, Shi HX. 2005. Relationship between trefoil factor 1 expression and gastric mucosa injuries and gastric cancer [J]. World J Gastroenterol, 11(17): 2674-2677.

Rio MC, Chenard MP, Wolf C, Marcellin L, Tomasetto C, Lathe R. 1991. Induction of $\mathrm{pS} 2$ and hSP genes as markers of mucosal ulceration of the digestive tract [J]. Gastroenterology, 100(2): 375-379.

Rodrigues S, Rodrigue CM, Attoub S, Flejou JF, Bruyneel E, Bracke M, Emami S, Gespach C. 2006. Induction of the adenoma-carcinoma progression and Cdc25A-B phosphatases by the trefoil factor TFF1 in human colon epithelial cells [J]. Oncogene, 25(50): 6628-6636.

Rodrigues S, Van Aken E, Van Bocxlaer S, Attoub S, Nguyen QD, Bruyneel E, Westley BR, May FEB, Thim L, Mareel M, Gespach C, Emami S. 
2003. Trefoil peptides as proangiogenic factors in vivo and in vitro: implication of cyclooxygenase-2 and EGF receptor signaling [J]. FASEB, 17(1): 7-16

Smid M, Wang Y, Klijn JGM, Sieuwerts AM, Zhang Y, Atkins D, Martens JWM, Foekens A. 2006. Genes associated with breast cancer metastatic to bone [J]. J Clin Oncol, 24(15): 2261-2267.

Takano T, Yamada H. 2009. Trefoil factor 3(TFF3): a promising indicator for diagnosing thyroid follicular carcinoma [J]. Endocr J, 56(1): 9-16

Taupin DR, Kinoshita K, Podolsky DK. 2000. Intestinal trefoil factor confers colonic epithelial resistance to apoptosis [J]. Proc Natl Acad Sci USA, 97(2): 799-804.

Taupin DR, Pedersen J, Familari M, Cook G, Yeomans N, Giraud AS. 2001. Augmented intestinal trefoil factor (TFF3) and loss of pS2(tff1) expression precedes metaplastic differentiation of gastric epithelium [J]. Lab Invest, 81(3): 397-408.

Taupin DR, Podolsky DK. 2003. Trefoil factors: initiators of mucosal healing [J]. Nat Rev Mol Cell Biol, 4(9): 721-732.

Theisinger B, Welter C, Seitz G, Rio MC, Lathe R, Chambon P. 1991. Expression of the breast cancer associated gene $\mathrm{pS} 2$ and the pancreatic spasmolytic polypeptide gene (hSP) in diffuse type of stomach carcinomas [J]. Eur J Cancer, 27(6): 770-773.

Thim L. 1989. A new family of growth factor-like peptides:árefoilô disulphide loop structures as a common feature in breast cancer associated peptide (pS2), pancreatic spasmolytic polypeptide (PSP) and frog skin peptides (spasmolysins) [J]. FEBS Lett, 250(1): 85-90.

Thim L, May FEB. 2005. Structure of mammalian trefoil factors and functional insights [J]. Cell Mol Life Sci, 62(24): 2956-2973.

Thuwajit P, Chawengrattanachot W, Thuwajit C, Sripa B, May FE, Wsstley BR, Tepsiri NN, Paupairoj A, Chau-In S. 2007. Increased TFF1 trefoil protein expression in Opisthorchis viverrini-associated cholangiocarcinoma is important for invasive promotion $[\mathrm{J}]$. Hepatol Res, 37(4): 295-304.

Vestergaard EM, Borre M, Poulsen SS, Nexo E, Torring N. 2006. Plasma levels of trefoil factors are increased in patients with advanced prostate cancer [J]. Clin Cancer Res, 12(3): 807-812.

Vestergaard EM, Nexo E, Wendt A, Guthmann. 2008. Trefoil factors in human milk [J]. Early Hum Dev, 84(10): 631-635.

Wong WM, Poulsom R, Wright NA. 1999 .Trefoil peptides [J]. Gut, 44(6): 890-895.

Wright NA, Pike C, Elia G. 1990a. Induction of a novel epidermal growth factor-secreting cell lineage by mucosal ulceration in human gastrointestinal stem cells [J]. Nature, 343(6253): 82-85.

Wright NA, Poulsom R, Stamp GW, Hall PA, Jeffery RE, Longcroft JM, Rio MC, Tomasetto C, Chambon P. 1990b. Epidermal growth factor (EGF/URO) induces expression of regulatory peptides in damaged human gastrointestinal tissues [J]. Pathol, 162(4): 279-284.

Wright NA, Poulsom R, Stamp G, Vannorden S, Sarraf C, Elia G. 1993. Trefoil peptide gene expression in gastrointestinal epithelial cells in inflammatory bowel disease [J]. Gastroenterology, 104(1): 12-20.

Yamachika T, Werther JL, Bodian C, Babyatsky M, Tatematsu M, Yamamura Y, Chen A, Itzkowitz S. 2002. Intestinal trefoil factor: a marker of poor prognosis in gastric carcinoma [J]. Clin Cancer Res, 8(5): 1092-1099.

Yio X, Zhang J, Babytsky M, Chen A, Lin J, Fan QX, Werther JL, Itzkowitz S. 2005. Trefoil factor-3 is associated with aggressive behavior of colon cancer cells [J]. Clin Exp Metastasis, 22(2): 157-165.

Zhang J, Zhang Y, Wan SG, Wei SS, Lee WH, Zhang Y. 2005. Bm-TFF2, a trefoil factor protein with platelet activation activity from frog Bombina maxima skin secretions [J]. Biochem Biophys Res Commun, 330(4): 1027-10

《动物学研究》近年发表文章在 2008 年中被引频次最高的前 43 篇文章题录

(数据由中国科技信息研究所提供)

\begin{tabular}{|c|c|c|}
\hline 第一作者 & 题 名 & 年, 卷期 \\
\hline 张云 & $\begin{array}{c}\text { 两栖类动物皮肤分泌物及其生物学适应意义う } \\
\text { 大蹼铃蟾皮肤分泌物蛋白质多肽组的启示 }\end{array}$ & $2006,27(1)$ \\
\hline 邵明勤 & 甘肃兴隆山自然保护区森林演替对鸟类群落结构的影响 & $2005,26(2)$ \\
\hline 周伟 & 同一生境牛蛙与昭觉林蛙蝌蚪的食性比较 & $2005,26(1)$ \\
\hline 朱晓琛 & 微卫星评价牙鲆雌核发育二倍体纯合性 & $2006,27(1)$ \\
\hline 全迎春 & 应用微卫星多态分析四个鲤鱼群体的遗传多样性 & $2005,26(6)$ \\
\hline 刘振生 & 贺兰山区马鹿对冬季生境的选择性 & $2004,25(5)$ \\
\hline 王彦平 & 惊飞距离--杭州常见鸟类对人为侵扰的适应性 & $2004,25(3)$ \\
\hline 张志伟 & 草鱼野生和养殖群体间遗传变异的微卫星分析 & $2006,27(2)$ \\
\hline 陶峰勇 & 中国大鲵四种群的遗传结构和地理分化 & $2005,26(2)$ \\
\hline 沈文英 & 饥饿对银鲫血液组分和卵巢发育的影响 & $2003,24(6)$ \\
\hline 赵平 & 崇明东滩冬季水鸟的种类和生境偏好 & $2003,24(5)$ \\
\hline 宁应之 & 甘肃天水麦积山风景名胜区土壤纤毛虫的物种多样性 & $2007,28(4)$ \\
\hline 叶朗惠 & 尼西鸡遗传多样性微卫星标记分析 & $2006,27(1)$ \\
\hline
\end{tabular}

